OPEN ACCESS

Edited by:

Lian Xiang Luo,

Guangdong Medical University, China

Reviewed by:

Xing Liang,

Stanford University, United States

Yanqing Liu,

Columbia University, United States

Ling Li,

University of North Dakota,

United States

*Correspondence:

Chuanjun Chen

ccj6318@sina.com.cn

Specialty section:

This article was submitted to

Human and Medical Genomics,

a section of the journal

Frontiers in Genetics

Received: 03 January 2022 Accepted: 07 February 2022

Published: 01 March 2022

Citation:

$L i T$, Wang $Y$, Xiang $X$ and Chen $C$

(2022) Development and Validation of

a Ferroptosis-Related IncRNAs

Prognosis Model in Oral Squamous

Cell Carcinoma.

Front. Genet. 13:847940

doi: 10.3389/fgene.2022.847940

\section{Development and Validation of a Ferroptosis-Related IncRNAs Prognosis Model in Oral Squamous Cell Carcinoma}

\author{
Tao $\mathrm{Li}^{1,2}$, Yi Wang ${ }^{1}$, Xianwang Xiang ${ }^{1}$ and Chuanjun Chen ${ }^{1 *}$ \\ ${ }^{1}$ Department of Oral and Maxillofacial Surgery, The First Affiliated Hospital of USTC, Division of Life Sciences and Medicine, \\ University of Science and Technology of China, Hefei, China, ${ }^{2}$ WanNan Medical College, Wuhu, China
}

Objectives: Ferroptosis is an iron-dependent form of programmed cell death, which affects the prognosis of many cancers. Some long non-coding RNA (IncRNA) can affect the prognosis of cancer by regulating the process of ferroptosis. However, the role of ferroptosis-related IncRNA (frlncRNA) in oral squamous cell carcinoma (OSCC) is not yet clear.

Materials and Methods: The data of OSCC patients were downed from The Cancer Genome Atlas (TCGA). After univariate and multivariate Cox regression analysis, the prognosis-related ferroptosis-related IncRNAs were obtained to construct a prognostic model. Calculated the risk score to divide patients into high and low risk groups, and evaluated the predictive ability of the model and the differential expression of immunity in the high and low risk groups.

Results: The prognostic model for OSCC was constructed based on 8 prognostic-related frlncRNAs which co-expressed with 25 mRNAs. Kaplan-Meier analyses displayed that the risk score is inversely proportional to patient survival. Receiver operating characteristic $(\mathrm{ROC})$ and decision curve analysis (DCA) indicated that the risk score is superior to other clinical characteristics, and independent prognostic analysis demonstated that risk score is independent factor for the overall survival (OS) rate. The results of immunological analysis showed differences in immune cells, functions, immune checkpoints, and m6A expression between high and low risk groups.

Conclusion: We constructed an OSCC patients prognosis model based on 8 frlncRNAs, which can provide prognostic evaluation and immune analysis for OSCC patients, and provided new direction for OSCC targeted therapy.

Keywords: ferroptosis, IncRNA, OSCC, immunity, prognosis model

\section{INTRODUCTION}

Oral cancer is one of the common malignant tumors. Recent global estimates show that there will be 377,713 new cases and 177,757 deaths from oral cancer in 2020 (Sung et al., 2021). OSCC is the most common, accounting for more than $90 \%$ of all oral cancers (Chi et al., 2015). Although treatment methods are constantly improving, the prognosis of OSCC is still poor, only about $50 \%$ in 5 years 


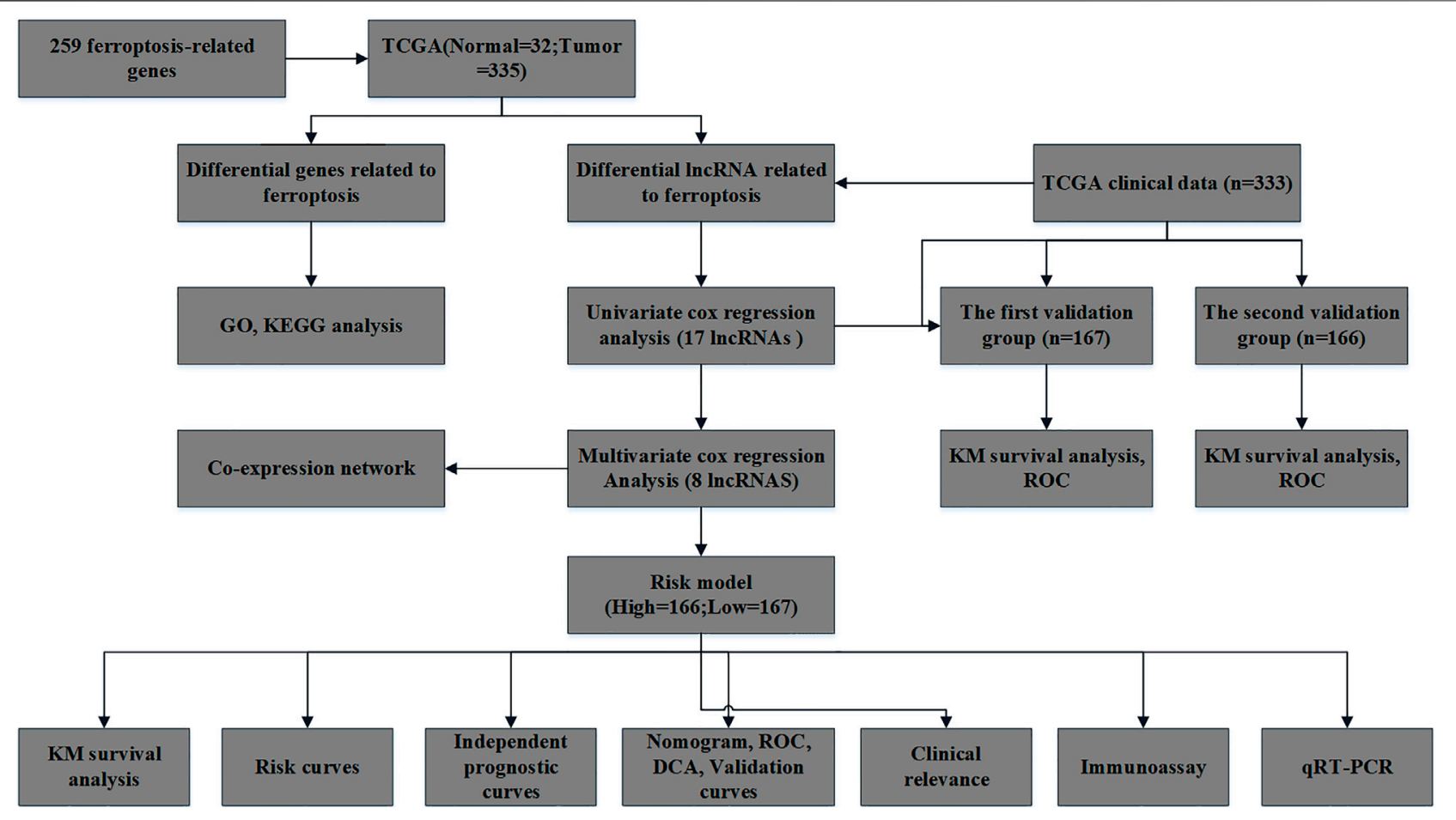

FIGURE 1 | The flowchart of this study.

(Kurihara-Shimomura et al., 2020). At present, the evaluation of prognosis and survival in OSCC is still based on the traditional TNM staging standard. However, due to the differences in the genetic signs of patients with the same TNM staging status, their response to treatment and individual differences may affect the prognosis assessment of patients with OSCC. Therefore, studying the biological, genetic and epigenetic changes of OSCC, especially the underlying mechanism of aggressive phenotype, is essential to improve the prognosis of OSCC patients. Studies have found that programmed cell death (PCD) is related to the occurrence, progression and metastasis of tumors (Lee et al., 2018). Ferroptosis is a new type of PCD, which is different from previous apoptosis and autophagy in its unique mechanism, that is, iron-dependent reactive oxygen species (ROS) accumulation and irresistible lipid metabolism. Oxidation leads to cell death (Dixon et al., 2012). It is well known that inducing cell death is a feasible cancer treatment. Ferroptosis has also been identified as a potential prevention or treatment strategy that triggers cancer cell death, especially for malignant tumors that are resistant to traditional therapies (Roh et al., 2016). Fukuda M et al. reported that ferroptosis plays an important role in oral cancer. Some genes that promote the proliferation of OSCC cells, such as GPX4 and SREBP, seem to protect cells from ferroptosis (Fukuda et al., 2021).

LncRNAs are non-protein coding genes larger than 200 nucleotides to distinguish them from small non-coding RNAs (Kopp and Mendell, 2018). In recent years, with the development of high-throughput sequencing technology, it has been discovered that a large number of non-coding genes play an important role in the occurrence and development of tumors (Gao et al., 2020). Studies have shown that lncRNAs are involved in cell growth, invasion and metastasis. It also plays an important role in OSCC, such as: IncRNACASC9 promotes autophagy apoptosis of OSCC cells by inhibiting the AKT/mTOR signaling pathway to increase autophagy (Yang et al., 2019); lncRNAUCA1 exerts its oncogene effect in OSCC cells through the UCA1/miR-184/SF1 axis (Fang et al., 2017).

The role and prognostic value of frlncRNAs in OSCC are currently unclear. Here, we screened frlncRNAs related to the prognosis of OSCC to construct a prognostic model and study its possible mechanism. Meanwhile, we also analyzed the functional enrichment analysis of differential genes, the differences in the expression of immune cell infiltration, immune checkpoint and m6A between high and low risk groups. It is hoped that new biomarkers can be provided for the targeted therapy of OSCC. The flow chart of this study is shown in Figure 1.

\section{MATERIALS AND METHODS}

\section{Data acquisition}

The RNA sequencing (RNA-seq) data of OSCC patients were downed from TCGA database on 2021.09.29, including 32 samples with normal RNA sequences, 335 samples with OSCC, In addition, we collected 333 cases corresponding clinical datas of tumor samples from TCGA. The clinical data included survival status, survival time, gender, age, tumor stage, pathological grade, $\mathrm{T}$ stage, $\mathrm{N}$ stage and $\mathrm{M}$ stage. 


\section{Identification of frIncRNAs}

The human GTF interpretation file were downloaded from Ensembl (http://asia.ensembl.org). 19,573 mRNAs and 14,056 lncRNAs were differentiated and extracted by operating the Strawberry Perl software. A total of 259 ferroptosis-related genes (Supplementary Table S1) included driver genes, suppressor genes and marker genes were extracted from the FerrDb database (http://www.zhounan.org/ ferrdb). The correlation between ferroptosis-related genes and all lncRNAs was analyzed through the $\mathrm{R}$ limma package to obtain frlncRNAs and ferroptosis-related mRNAs(frmRNAs). The filter standard was set that correlation coefficient $>0.4$ and $p$-value $<0$. 001, respectively.

\section{Screening Differentially Expressed Genes and Enrichment Analysis}

The "limma" package of $\mathrm{R}$ software was used to screen of differentially expressed genes (DEG) related to ferroptosis between the normal group and the tumor group. The standard is the false discovery rate $(\mathrm{FDR})<0.05$ and $|\log \mathrm{FC}|>1$. The packages of "colorspace," "stringi" and "colorspace" were to perform Gene Ontology (GO) analysis and Kyoto Encyclopedia of Genes and Genomes (KEGG) pathway analysis on differentially expressed genes, with $p$-value $<0.05$ and $Q$-value $<0.05$ as the screening conditions.

\section{Construction and Evaluation of the Prognostic Model of frlncRNAs}

The most prognostic frlncRNAs were screened by univariate and multivariate COX regression analysis. Over and above that, patients were classified into low-risk ( $<$ median) or high-risk ( $>$ median) groups according to the median of risk score. The risk scores of OSCC parents were calculated on the basis of the following formula. $\mathrm{N}$ represents the finally optioned lncRNA.

$$
\begin{aligned}
\text { Risk score }= & \operatorname{Exp}_{\text {lncRNA1 }} \times \beta_{\text {lncRNA1 }}+\operatorname{Exp}_{\text {lncRNA2 }} \times \beta_{\text {lncRNA2 }}+\cdots \\
& +\operatorname{Exp}_{\text {lncRNAn }} \times \beta_{\text {lncRNAn }}
\end{aligned}
$$

The Kaplan-Meier (K-M) survival curves was used to compare the overall survival (OS) between high-risk and low-risk group, receiver operating characteristic (ROC) curves and decision curve analysis (DCA) were used to evaluate whether the predictive power of risk score was better than that of other clinical characteristics. Univariate and multivariate COX regression analyses were used to determine whether risk scores was independent of other clinical characteristics as a prognostic factor in patients with OSCC. Finally, all independent prognostic parameters were incorporated into the construction of the nomogram to predict the 1,2 , and 3 years overall survival of patients. The accuracy of the nomogram's predictions was assessed by the calibration curve.

\section{Internal Validation and Clinical Relevance Analysis}

The 333 cliniacal data were randomly divided into two groups to validate the predictive ability of the model according to the ratio of 1:1 through the package "caret" of R (Table 1). In order to compare differences in clinical characteristics between high and low risk groups, the clinical data were divided into several subgroups. Moreover, we also compared the differences in the expression of frlncRNAs in various clinical features.

\section{Establishment of a Co-expression Network of IncRNA-mRNA Related to Ferroptosis}

Investigated the relationship between frlncRNAs and frmRNAs, and constructed an IncRNA-mRNA co-expression network through Cytoscape.

\section{Immunoassay}

Immune cell infiltration files were downloaded from (http:// timer.cistrome.org).The difference between immune cells in the high and low risk groups were evaluated by the TIMER, CIBERSORT, CIBERSORT-ABS, QUANTISEQ, MCPCOUNTER, $X C E L L$ and EPIC. In addition, we also compared the differences in immune function, immune checkpoint and m6A between high and low risk groups.

\section{RNA Extraction and Quantitative PCR}

There were four pairs of OSCC and adjacent samples collected from the First Affiliated Hospital of University of Science and Technology of China, and stored in liquid nitrogen at $-196^{\circ} \mathrm{C}$. According to the manufacturer's instructions, total RNA was extracted by Trizol reagent (Yisheng Biotechnology, China), cDNA was synthesized by HiScript II 1st Strand cDNA Synthesis Kit (+gDNA wiper) (Vazyme, China), Hieff ${ }^{\circledast}$ qPCR SYBR Green Master Mix (Low Rox) (Yisheng Biotechnology, China) was used for amplification, GAPDH was set as an endogenous control. we selected 4 lncRNAs (STARD4-AS1, MIAT, AC099850.3, AL512274.1) of the model for $\mathrm{qPCR}$. The relative quantification method of $2^{-\Delta \Delta C T}$ was used to normalize the expression of lncRNA. Each group was repeated three times and the mean value was used for analysis. All patients gave informed consent and signed an informed consent form. The primer sequences of these four lncRNAs are provided in Supplementary Table S2.

\section{Statistical Analysis}

All data were analyzed using Rstudio or SPSS 20.0. Paired samples were used by independent $t$-test or one-way analysis of variance. Kaplan-Meier survival analysis was used to assess the difference in survival between the high and low risk groups of OSCC patient prognosis model based on frlncRNAs. ROC and DCA curves were used to evaluate the predictive performance of OSCC prognostic model. The statistical significance was set at $p<0.05$.

\section{RESULTS}

\section{Enrichment Analysis of Differential mRNAs Related to Ferroptosis}

We found 62 differential mRNAs related to ferroptosis (17 downregulated and 45 up-regulated) (Supplementary Table S3); BP participated in response to oxidative stress and cellular response to 
TABLE 1 | The clinical datas in different groups.

\begin{tabular}{|c|c|c|c|}
\hline \multirow[t]{2}{*}{ Variables } & \multirow{2}{*}{$\frac{\text { TCGA(Total) }}{(n=333)}$} & \multicolumn{2}{|c|}{ Validation } \\
\hline & & $\begin{array}{l}\text { The first validation } \\
\text { group }(n=167)\end{array}$ & $\begin{array}{c}\text { The second validation } \\
\text { group }(n=166)\end{array}$ \\
\hline \multicolumn{4}{|l|}{ Age (\%) } \\
\hline$<=65$ & 210 (63.06) & 108 (64.67) & $102(61.45)$ \\
\hline$>65$ & $123(36.94)$ & 59 (35.33) & 64 (38.55) \\
\hline \multicolumn{4}{|l|}{ Gender (\%) } \\
\hline FEMALE & 100 (30.03) & 55 (32.93) & $45(27.11)$ \\
\hline MALE & 233 (69.07) & 112 (67.07) & 121 (72.89) \\
\hline \multicolumn{4}{|l|}{ Grade (\%) } \\
\hline G1-2 & 257 (77.18) & 127 (76.05) & $130(78.31)$ \\
\hline G3-4 & 67 (20.12) & 36 (21.55) & 31 (18.67) \\
\hline $\mathrm{GX}+$ unknow & $9(2.70)$ & $4(2.40)$ & 5 (3.01) \\
\hline \multicolumn{4}{|l|}{ Stage(\%) } \\
\hline Stagel-II & 75 (22.52) & 40 (23.95) & 35 (21.08) \\
\hline StagellI-IV & $223(66.97)$ & 108 (64.67) & 115 (69.28) \\
\hline unknow & $35(10.51)$ & 19 (11.38) & $16(9.64)$ \\
\hline \multicolumn{4}{|l|}{$\mathrm{T}(\%)$} \\
\hline $\mathrm{T} 1-\mathrm{T} 2$ & $134(40.24)$ & 66 (39.52) & 68 (40.96) \\
\hline T3-T4 & $172(51.65)$ & $86(51.50)$ & $86(51.81)$ \\
\hline TX + unknow & $27(8.11)$ & $15(8.98)$ & $12(7.23)$ \\
\hline \multicolumn{4}{|l|}{$\mathrm{M}(\%)$} \\
\hline $\mathrm{MO}$ & 121 (36.34) & 65 (38.92) & 56 (33.73) \\
\hline$M X+$ unknow & $212(63.66)$ & $102(61.08)$ & $110(66.27)$ \\
\hline \multicolumn{4}{|l|}{ N (\%) } \\
\hline NO & 120 (36.04) & 63 (37.72) & 57 (34.34) \\
\hline $\mathrm{N} 1-3$ & $158(47.45)$ & 73 (43.71) & $85(51.20)$ \\
\hline $\mathrm{NX}+$ unknow & 55 (16.51) & 31 (18.56) & $24(14.46)$ \\
\hline
\end{tabular}

chemical stress; $\mathrm{MF}$ regulated organic anion transmembrane transporter activity; CC participated in basal plasma membrane,basal part of cell and apical part of cell (Figure 2A). KEGG analysis results demonstated that overexpressed genes were mainly involved in MicroRNAs in cancer, HIF-1 signaling pathway, Ferroptosis, Fluid shear stress and atherosclerosis, Kaposi sarcoma-associated herpesvirus infection, mTOR signaling pathway, Cysteine and methionine metabolism, Central carbon metabolism in cancer, Biosynthesis of amino acids and Pancreatic cancer (Figure 2B).

\section{Prognostic-Related frlncRNAs Risk Model and Co-expression Network}

A total of 377 differential frlncRNAs were identified, which of 17 were associated with prognosis after univariate analysis $(p<0.05)$ (Supplementary Table S4; Figure 2C). Multivariate COX regression analysis was performed to select the optimal prognostic frlncRNAs, according to Akaike Information Criterion (AIC), finally 8 frlncRNAs were used to construct the OSCC prognostic model (Table 2), and patients were divided into high-risk groups $(n=166)$ and low-risk groups $(n=167)$ based on the median risk score. A total of 25 frmRNAs were co-expressed with these 8 frlncRNAs, of which 6 were co-expressed with AC099850.3 (Supplementary Table S5; Figure 2D).

\section{The evaluation of Prognosis Model}

In independent prognostic analysis, the univariate and multivariate COX analysis showed the risk score of frlncRNAs (HR: 1.721, 95CI 1.438-2.059), age (HR: 1.037 95CI: 1.020-1.055) and tumor stage
(HR: 1.595, 95CI: 1.279-1.988) were independent prognostic factors of OS in patients with OSCC $(p<0.05$, Figures 3A,B). There were more deaths can be observed in the high-risk group from Figures 3C,D. We as well observed that STARD4-AS1, MIAT and AL512274.1 were more expressed in the low-risk group; on the contrary, AC099850.3, AC090246.1, ALMS1-IT1, AC021087.4, and HOTARM1 were more expressed in the high-risk group (Figure 3E). In addition, as shown in Figure 4A, Kaplan-Meier analysis illustrated that the expression of high-risk lncRNAs characteristics was significantly different from the low-risk group in OS $(p<0.001)$, and the patients' risk score was inversely proportional to the survival rate of OSCC patients. It can be seen that there are some numbers under the Figure 4A. For e.g., when the time node is 1 , the number corresponding to red is 110 , which represents the number of people who survived in the high-risk group when the follow-up time is 1 year. We assessed the sensitivity and specificity of the risk model through AUC (the area under the ROC curve), the AUC of the model to predict 1, 2, and 3 years OS was $0.690,0.672$, and 0.677 , respectively (Figure 4B). And the ROC (Figure 4C) and DCA (Figure 4D) curves of the risk score of frlncRNAs indicated that risk score was better than traditional clinical pathology features in predicting the prognosis of OSCC. We included the independent factors of age, tumor stage, and risk score in the multivariate independent prognostic analysis into the nomogram (Figure 4E) to predict 1, 2, and 3 years survival, and used the calibration curve to verify. The results (Figures $4 \mathbf{F}-\mathbf{H}$ ) displayed that the calibration curves were close to the ideal slope, which meaned that age, tumor, and risk scores together can accurately predict 1-, 3-, and 5 years OS of patients. 

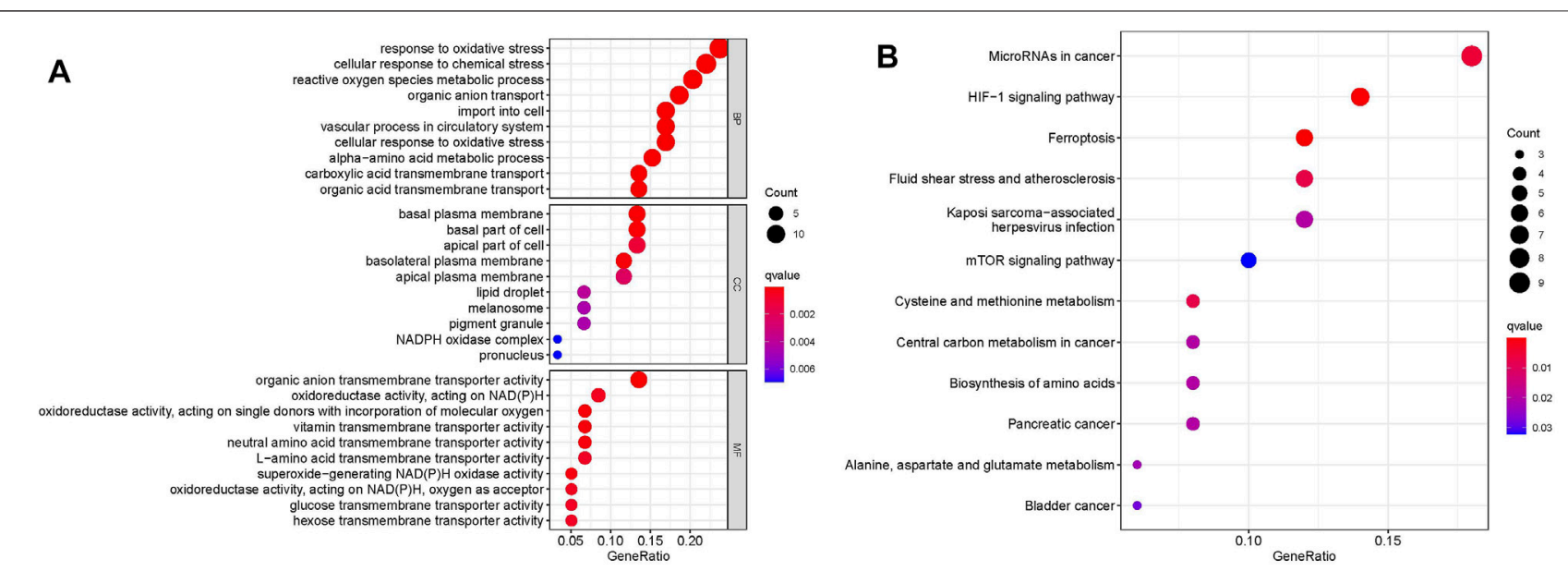

C

$\begin{array}{lrr} & \text { pvalue } & \begin{array}{r}\text { Hazard ratio } \\ \text { STARD4-AS1 }\end{array} \\ 0.047 & 0.466(0.219-0.990) \\ \text { AC099850.3 } & 0.002 & 1.034(1.012-1.056) \\ \text { AC090246.1 } & 0.041 & 1.317(1.011-1.717) \\ \text { DDX59-AS1 } & 0.020 & 1.615(1.080-2.414) \\ \text { LINC00942 } & 0.034 & 1.027(1.002-1.052) \\ \text { ALMS1-IT1 } & 0.004 & 2.066(1.261-3.386) \\ \text { AC021087.4 } & 0.048 & 1.123(1.001-1.259) \\ \text { AL132712.1 } & 0.030 & 1.123(1.011-1.246) \\ \text { SLC16A1-AS1 } & 0.047 & 1.918(1.007-3.650) \\ \text { AC002116.2 } & 0.038 & 1.305(1.015-1.676) \\ \text { MIAT } & 0.048 & 0.826(0.683-0.998) \\ \text { HOTAIRM1 } & 0.031 & 1.055(1.005-1.107) \\ \text { LINC02560 } & 0.048 & 0.980(0.961-1.000) \\ \text { KLHL7-DT } & 0.002 & 1.718(1.217-2.423) \\ \text { AC104041.1 } & 0.040 & 1.269(1.010-1.594) \\ \text { AL512274.1 } & 0.017 & 0.962(0.931-0.993) \\ \text { AC092115.3 } & 0.044 & 1.611(1.014-2.560)\end{array}$

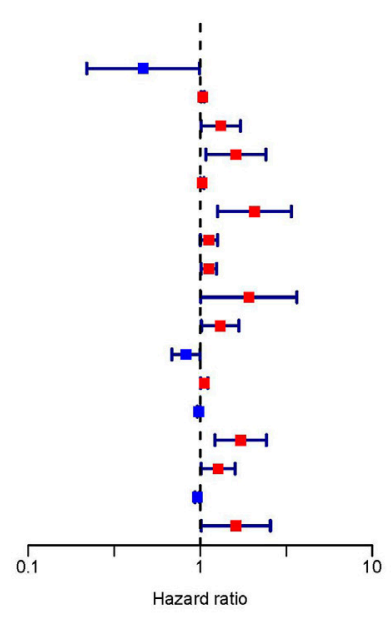

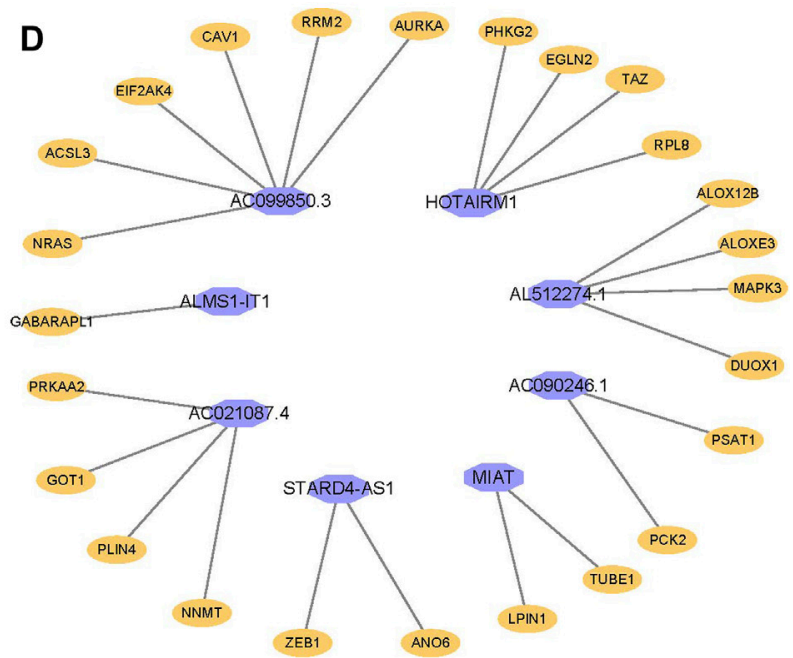

FIGURE 2 | (A) Go enrichment analysis. (B) KEGG enrichment analysis. (C) Forest plot represents 17 IncRNAs related to OSSC prognosis obtained by univariate analysis. (D) The prognostic-related IncRNAs and their co-expressed mRNA obtained from multivariate COX regression analysis, purple represents IncRNA, orange represents $\mathrm{mRNA}$.

TABLE 2 | 8 frlncRNAs by multivariate Cox regression analysis.

\begin{tabular}{lccccc}
\hline LncRNA & Coef & HR & HR.95L & HR.95H & p-value \\
\hline STARD4-AS1 & -0.559 & 0.572 & 0.270 & 1.212 & 0.145 \\
AC099850.3 & 0.028 & 1.029 & 1.007 & 1.052 & 0.011 \\
AC090246.1 & 0.362 & 1.436 & 1.051 & 1.962 & 0.023 \\
ALMS1-IT1 & 0.557 & 1.746 & 1.034 & 2.947 & 0.037 \\
AC021087.4 & 0.140 & 1.151 & 1.027 & 1.288 & 0.016 \\
MIAT & -0.238 & 0.788 & 0.640 & 0.970 & 0.024 \\
HOTAIRM1 & 0.047 & 1.050 & 0.995 & 1.106 & 0.079 \\
AL512274.1 & -0.038 & 0.962 & 0.931 & 0.996 & 0.030
\end{tabular}

\section{The clinical Relevance Analysis and Internal Validation}

The clinical variables were divided into several subgroups. We compared the differences in the OS of patients in the high and low risk groups in various clinical variables through Kaplan-Meier analysis. It can be seen that the OS of patients in the high risk group is lower in all subgroups including (age, gender, grade, stage, Tstage, $\mathrm{N}$ stage, $\mathrm{M}$ stage; $p<0.5$, Figures $5 \mathbf{A}-\mathbf{M}$ ). Furthermore, The results of the Kaplan-Meier analysis of the first validation group and the second validation group illustrated that the OS of the high-risk group was lower $(p<0.5$, Figures $5 \mathbf{N}, \mathbf{P})$, and the AUC of the risk score in both groups was greater than that of other clinical characteristics, which indicated that the predictive performance of the risk model was good (Figures 5O,Q). We also analyzed the differences in the expression of frlncRNAs among different clinical features(Figures 6A-F). It can be observed that ALMS1-IT1 was differentially expressed in males and females (Figure 6B). In addition, its expression is differential in $\mathrm{N}$ stage (Figure 6D); AL512274.1 had differences in the expression of grade, stage, and N stage (Figures 6C,D,F); AC099850.3 was differentially expressed on different grades (Figure 6C).

Immunity and Gene Expression and qPCR The immune response heatmap based on TIMER, CIBERSORT, CIBERSORT-ABS, QUANTISEQ, MCPCOUNTER, XCELL, and 


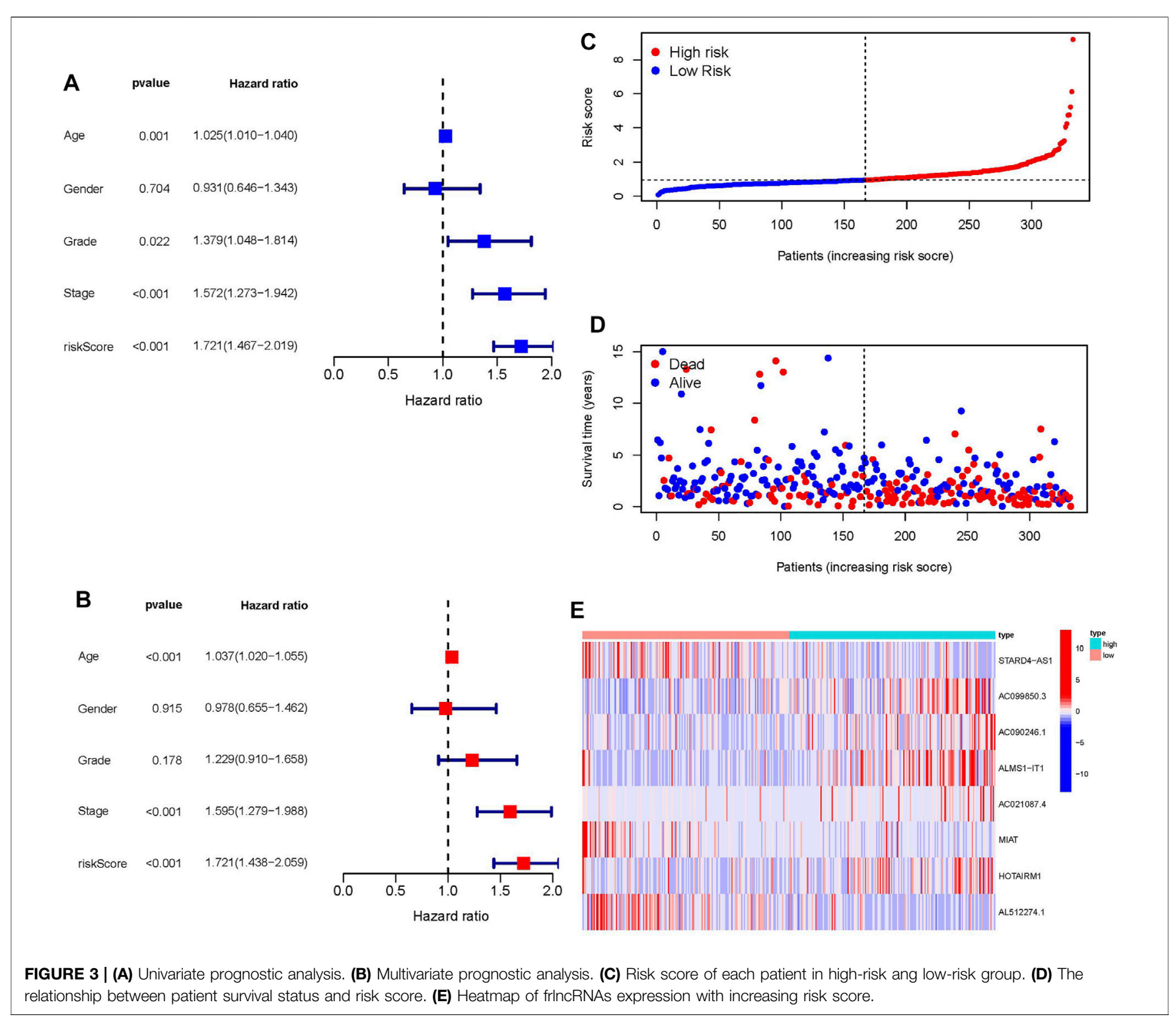

EPIC algorithms is shown in the Figure 6G. Analysis of differences in immune function based on TCGA-OSCC data showed that APC_co_inhibition, CCR, Check-point, Cytolytic_activity, HLA, Inflammation-promoting, Parainflammation, T_cell_co-inhibition, T_cell_co-stimulation and Type_II_IFN_Reponse were all highly expressed in the low-risk group (Figure 7A). At the same time, the comparison of m6A-related gene expression in high and low groups suggested that the expressions of WTAP, METTL14, YTHDF1, HNRNPC, YTHDC2, RBM15 and ALKBH5 were different $(p<0.05$, Figure 7B). In view of the importance of checkpoint inhibitor-based immunotherapy, we further explored the differences in immune checkpoint expression between the two groups. The results demonstrated that between the two groups CD48, TNFRSF9, CD40LG, CD160, CTLA4, KIR3DL1, CD200R1, CD28, PDCD1, ADORA2A, CD27, TIGIT, TNFRSF4, BTLA, ICOS, CD244, and IDO2 were expressed higher in the low-risk
group;CD70, CD276, TNFSF9 were the opposite $(p<0.05$, Figure 7C). As shown in Figures 7D-G, MIAT, AL512274.1 and STARD4-AS1 were more highly expressed in adjacent tissues than tumor, and AC099850.3 was highly expressed in tumor tissues, which is consistent with our model.

\section{DISCUSSION}

Ferroptosis is an iron-dependent cell death program, which has been shown to be related to tumor development and response to anti-tumor therapy (Chen et al., 2021a). LncRNA is an active participant in the immune regulation of 33 cancer types ( $\mathrm{Li}$ et al., 2020a). Morever, many lncRNAs are involved in the progression of malignant tumors and tumor resistance, and have become new biomarkers and therapeutic targets in cancer diagnosis and treatment (Wang et al., 2019a). The excellent predictive ability 


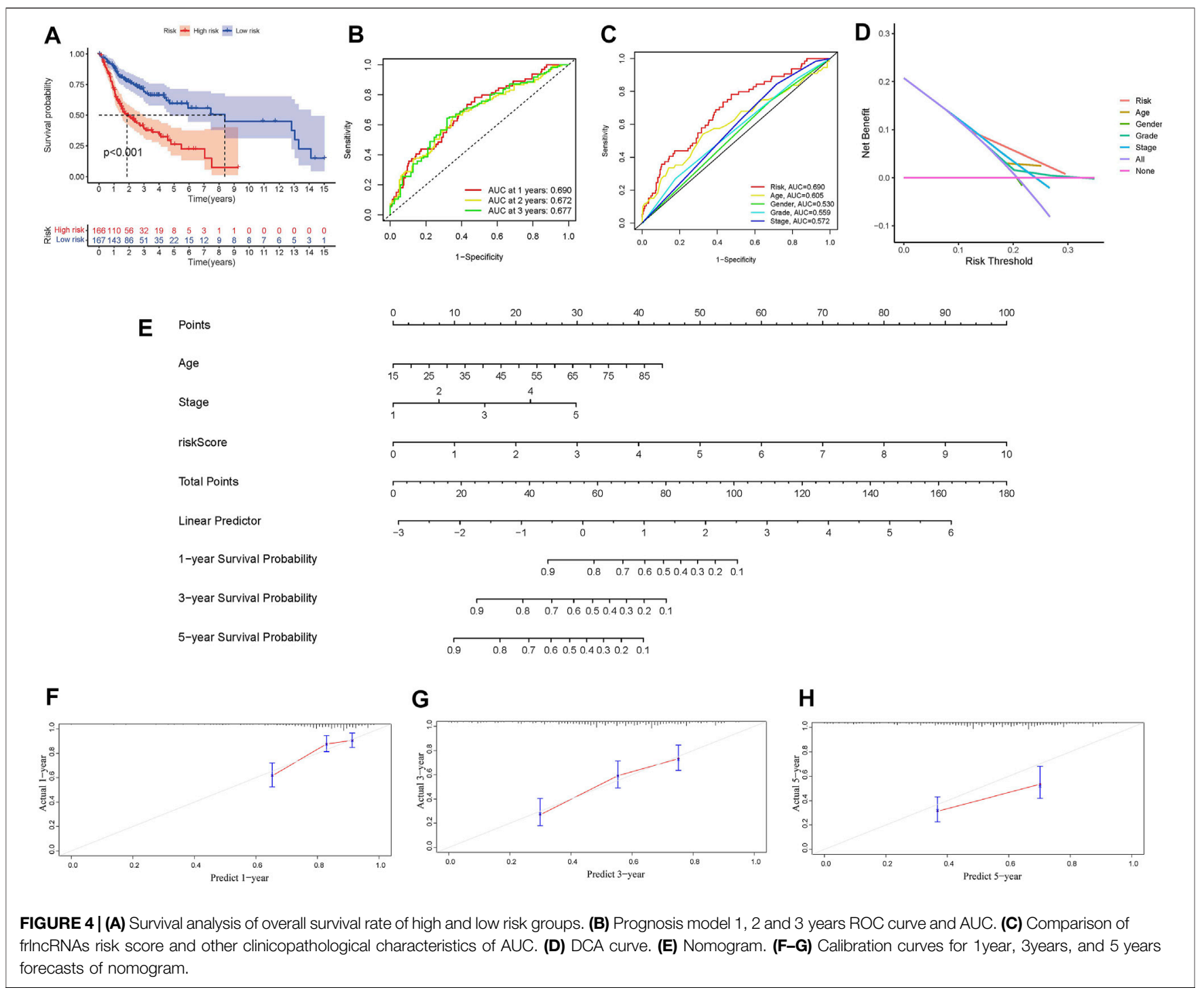

of frlncRNAs in cancer has been confirmed in a variety of cancers (Chen et al., 2021b; Lu et al., 2021; Jin et al., 2021; Li et al., 2021). However, the mechanism of frlncRNAs in OSCC is still unclear. Therefore, we conducted research on the correlation between frlncRNAs and the prognosis of OSSC. Based on 8 frlncRNAs, we constructed an OSSC risk prognosis model and divided patients into high and low risk groups according to risk scores. ROC, DCA, independent prognostic analysis to verify its predictive ability, the results showed that its predictive ability is better than other clinical features. These 8 frlncRNAs contained 5 risk factors: AC099850.3, AC090246.1, ALMS1-IT1, AC021087.4, and HOTARM1; three protection factors: STARD4-AS1, MIAT and AL512274.1. Among them, AC099850.3 is the most co-expressed lncRNA, which is related to six differently expressed mRNAs, namely (CAV1, NRAS, ACSL3, AURKA, EIF2AK4 and RRM2), and its high expression level is closely related to the reduction in the survival rate of patients with tongue cancer (Zhou et al., 2019). The high expression of ALMS1-IT1 can lead to poor prognosis of many cancers, such as head and neck squamous cell carcinoma (Xing et al., 2019), small cell lung cancer (Luan et al., 2021). The mechanism of its regulation is not yet fully understood. There are studies have shown that in small cell lung cancer, ALMS1-IT1 regulates AVL9 by adsorbing miRNAs, and participates in the regulation of cell cycle-related CDK pathways, thereby affecting tumor progression (Luan et al., 2021). HOTAIRM1 plays different roles in different diseases. It promotes autophagy and proliferation of acute myeloid leukemia cells with mutant nucleophosphoprotein by regulating the expression of EGR1 and ULK3 (Jing et al., 2021). HOTAIRM1 is down-regulated in liver cancer. The specific mechanism may be related to inhibiting the Wnt pathway to inhibit the proliferation of hepatocellular carcinoma cells and promote their apoptosis, thereby inhibiting the progression of liver cancer (Zhang et al., 2018). It is worth noting that as an oncogene, MIAT can proliferate and migrate in various cancer cells such as hepatocellular carcinoma (Huang et al., 2018), osteosarcoma (Zhang et al., 2019), and papillary thyroid carcinoma (Wang et al., 2019b). However, in our study, single-factor and multi- 


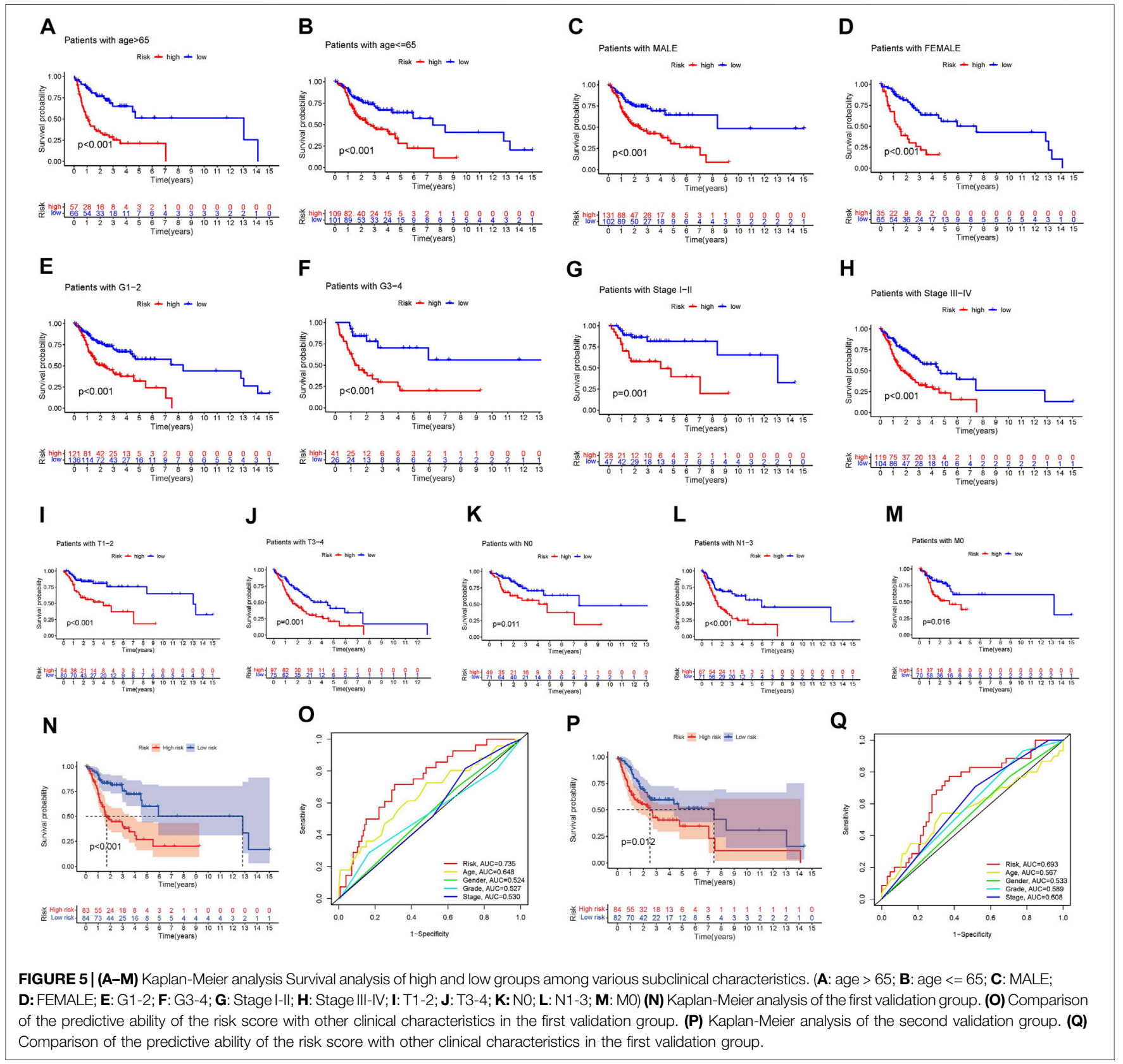

factor COX regression analysis showed that MIAT is a protective factor for the prognosis of OSCC. Furthermore, as shown in Figure 7E, MIAT expressed higher in adjacent tissues, which is consistent with our model. The role of AL512274.1 in cancer is still unclear, but studies have found that its co-expressed mRNA (MAPK3) is involved in the control of cell proliferation, differentiation and autophagy (Cagnol and Chambard, 2010; Jiang et al., 2021). STARD4-AS1, AC090246.1, AC021087.4 have not seen relevant studies in oral cancer and other tumors, and the specific mechanism is worthy of further investigation.

Considering that immunotherapy is playing an increasingly important role in cancer treatment. We compared the differences in immunity between high and low risk groups. As the Figure 7C displays that, except for CD70, CD276 and TNFSF9, almost all other differential genes in the immune checkpoint were expressed in the low-risk group, while m6A except YTHDC2 were mainly expressed in the high-risk group. Studies have reported that CD70 is highly expressed in oral cancer, and its specific CAR$\mathrm{T}$ cells can specifically recognize and effectively eliminate CD70positive HNSCC cells (Park et al., 2018). In addition, CD276 is highly expressed in head and neck squamous cell carcinoma and the blockade of CD276 significantly inhibited the lymph node metastasis of head and neck squamous cell carcinoma, which is one of the most challenging problems in the treatment of head and neck squamous cell carcinoma, indicating that targeting 


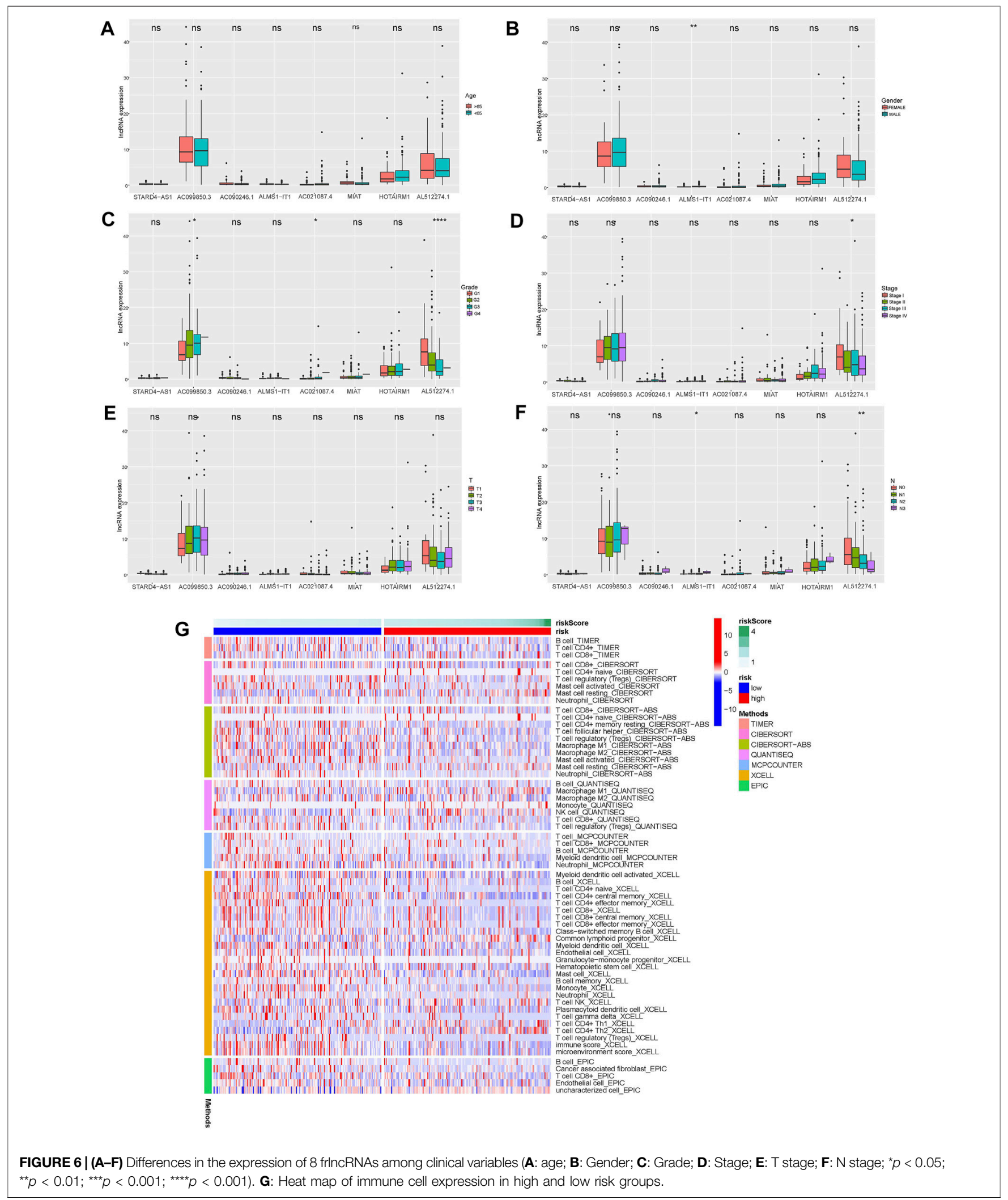




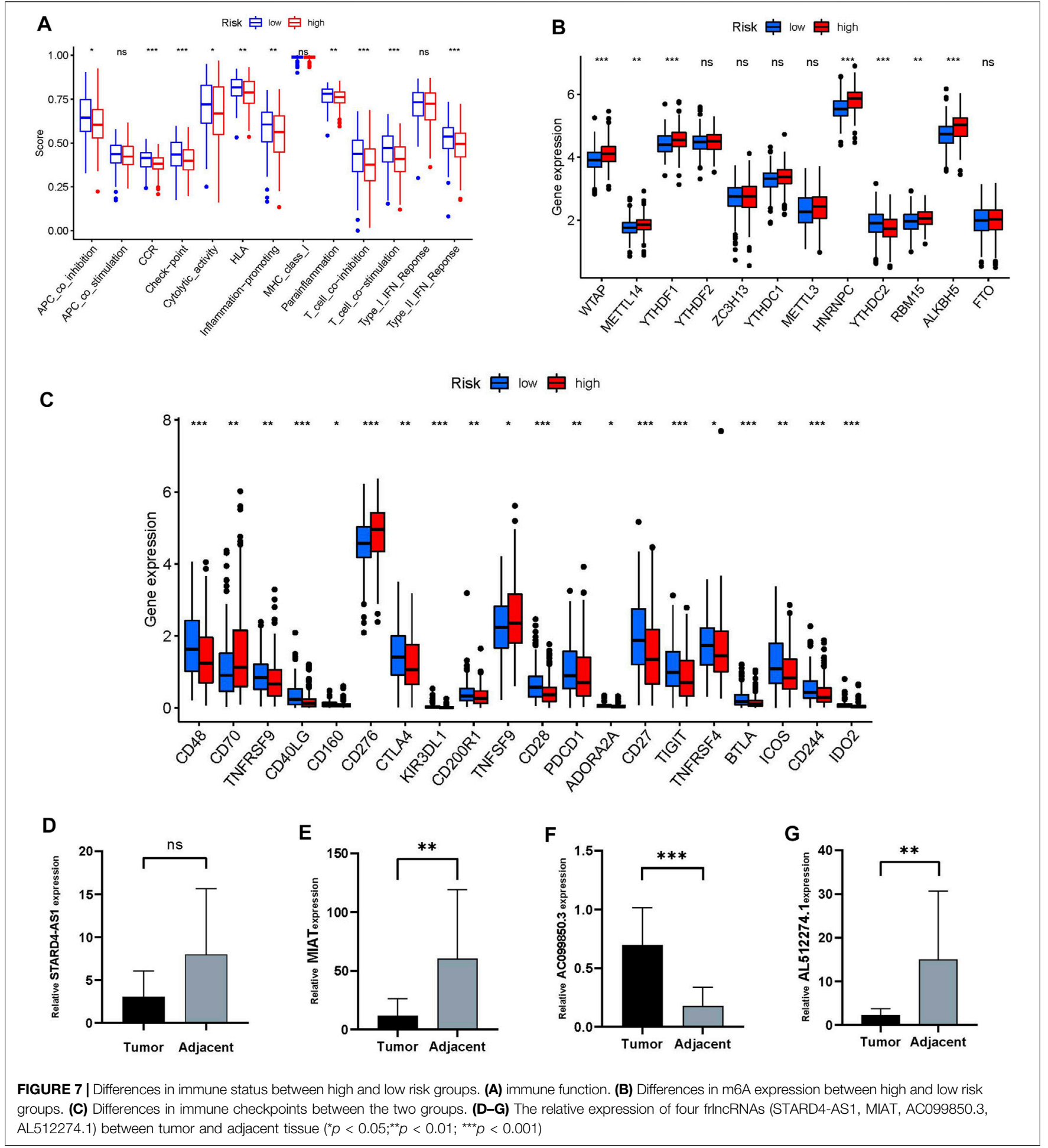

CD276 can enhance anti-tumor immunity (Wang et al., 2021). There are no studies on TNFSF9 and YTHDC2 in oral cancer. Yang Li reported that YTHDC2 is a tumor suppressor gene in the head and neck, which is highly expressed in normal tissues but low in tumors (Li et al., 2020b). In general, frlncRNAs have shown excellent performance in our research and are expected to become new biomarkers for the treatment of OSCC. However, there are some deficiencies in this study. First, the establishment and validation of the risk model is based on the TCGA database and its grouping, and there is a lack of external validation to provide more evidence for evaluating its clinical utility. Second, the number of experimental validations is limited, and the role 
and mechanism of most frlncRNAs in the progression of OSCC in this study are not clear, and further investigation is needed.

\section{CONCLUSION}

We constructed an OSCC patients prognosis model based on 8 frlncRNAs, which can provide prognostic evaluation and immune analysis for OSCC patients, and provided new direction for OSCC targeted therapy.

\section{DATA AVAILABILITY STATEMENT}

The original contributions presented in the study are included in the article/Supplementary Material, further inquiries can be directed to the corresponding author.

\section{ETHICS STATEMENT}

The studies involving human participants were reviewed and approved by the Ethics Committee in Medical Research of the First Affiliated Hospital of University of Science and Technology of China First Affiliated Hospital of University of

\section{REFERENCES}

Cagnol, S., and Chambard, J.-C. (2010). ERK and Cell Death: Mechanisms of ERKInduced Cell Death - Apoptosis, Autophagy and Senescence. FEBS J. 277 (1), 2-21. doi:10.1111/j.1742-4658.2009.07366.x

Chen, X., Kang, R., Kroemer, G., and Tang, D. (2021). Broadening Horizons: the Role of Ferroptosis in Cancer. Nature Reviews. Nat. Rev. Clin. Oncol. 18 (5), 280-296. doi:10.1038/s41571-020-00462-0

Chen, Z.-A., Tian, H., Yao, D.-M., Zhang, Y., Feng, Z.-J., and Yang, C.-J. (2021). Identification of a Ferroptosis-Related Signature Model Including mRNAs and lncRNAs for Predicting Prognosis and Immune Activity in Hepatocellular Carcinoma. Front. Oncol. 11, 738477. doi:10.3389/fonc.2021.738477

Chi, A. C., Day, T. A., and Neville, B. W. (2015). Oral Cavity and Oropharyngeal Squamous Cell Carcinoma-An Update. CA: a Cancer J. clinicians 65 (5), 401-421. doi:10.3322/caac. 21293

Dixon, S. J., Lemberg, K. M., Lamprecht, M. R., Skouta, R., Zaitsev, E. M., Gleason, C. E., et al. (2012). Ferroptosis: an Iron-dependent Form of Nonapoptotic Cell Death. Cell 149 (5), 1060-1072. doi:10.1016/j.cell.2012.03.042

Fang, Z., Zhao, J., Xie, W., Sun, Q., Wang, H., and Qiao, B. (2017). LncRNA UCA1 Promotes Proliferation and Cisplatin Resistance of Oral Squamous Cell Carcinoma by Sunppressing miR-184 Expression. Cancer Med. 6 (12), 2897-2908. doi:10.1002/cam4.1253

Fukuda, M., Ogasawara, Y., Hayashi, H., Okuyama, A., Shiono, J., Inoue, K., et al. (2021). Down-regulation of Glutathione Peroxidase 4 in Oral Cancer Inhibits Tumor Growth through SREBP1 Signaling. Anticancer Res. 41 (4), 1785-1792. doi:10.21873/anticanres.14944

Gao, N., Li, Y., Li, J., Gao, Z., Yang, Z., Li, Y., et al. (2020). Long Non-coding RNAs: The Regulatory Mechanisms, Research Strategies, and Future Directions in Cancers. Front. Oncol. 10, 598817. doi:10.3389/fonc.2020.598817

Huang, X., Gao, Y., Qin, J., and Lu, S. (2018). IncRNA MIAT Promotes Proliferation and Invasion of HCC Cells via Sponging miR-214. Am. J. Physiology-Gastrointestinal Liver Physiol. 314 (5), G559-G565. doi:10. 1152/ajpgi.00242.2017

Jiang, Q., Xue, D., Shi, F., and Qiu, J. (2021). Prognostic Significance of an Autophagy-Related Long Non-coding RNA Signature in Patients with Oral
Science and Technology of China. The patients/participants provided their written informed consent to participate in this study.

\section{AUTHOR CONTRIBUTIONS}

TL conceived the project, designed the research and drafted the manuscript. CC directed this research. TL and XX collected public data and specimens, analyzed, and tested. $\mathrm{CC}$ and YW revised the manuscript. All authors reviewed the manuscript.

\section{FUNDING}

This work was supported by the Fundamental Research Funds for the Central Universities (No. WK9110000146).

\section{SUPPLEMENTARY MATERIAL}

The Supplementary Material for this article can be found online at: https://www.frontiersin.org/articles/10.3389/fgene.2022.847940/ full\#supplementary-material

and Oropharyngeal Squamous Cell Carcinoma. Oncol. Lett. 21 (1), 29. doi:10. 3892/ol.2020.12290

Jin, Y., Wang, Z., He, D., Zhu, Y., Gong, L., Xiao, M., et al. (2021). Analysis of Ferroptosis-Mediated Modification Patterns and Tumor Immune Microenvironment Characterization in Uveal Melanoma. Front. Cell Dev. Biol. 9, 685120. doi:10.3389/fcell.2021.685120

Jing, Y., Jiang, X., Lei, L., Peng, M., Ren, J., Xiao, Q., et al. (2021). Mutant NPM1Regulated lncRNA HOTAIRM1 Promotes Leukemia Cell Autophagy and Proliferation by Targeting EGR1 and ULK3. J. Exp. Clin. Cancer Res. 40 (1), 312. doi:10.1186/s13046-021-02122-2

Kopp, F., and Mendell, J. T. (2018). Functional Classification and Experimental Dissection of Long Noncoding RNAs. Cell 172 (3), 393-407. doi:10.1016/j.cell. 2018.01.011

Kurihara-Shimomura, M., Sasahira, T., Shimomura, H., and Kirita, T. (2020). Peroxidan Plays a Tumor-Promoting Role in Oral Squamous Cell Carcinoma. Ijms 21 (15), 5416. doi:10.3390/ijms21155416

Lee, S. Y., Ju, M. K., Jeon, H. M., Jeong, E. K., Lee, Y. J., Kim, C. H., et al. (2018). Regulation of Tumor Progression by Programmed Necrosis. Oxid Med. Cell Longev 2018, 3537471. doi:10.1155/2018/3537471

Li, H., Liu, L., Huang, T., Jin, M., Zheng, Z., Zhang, H., et al. (2021). Establishment of a Novel Ferroptosis-Related lncRNA Pair Prognostic Model in colon Adenocarcinoma. Aging 13 (19), 23072-23095. doi:10.18632/aging.203599

Li, Y., Jiang, T., Zhou, W., Li, J., Li, X., Wang, Q., et al. (2020). Pan-cancer Characterization of Immune-Related lncRNAs Identifies Potential Oncogenic Biomarkers. Nat. Commun. 11 (1), 1000. doi:10.1038/s41467-020-14802-2

Li, Y., Zheng, J.-N., Wang, E.-H., Gong, C.-J., Lan, K.-F., and Ding, X. (2020). The m6A Reader Protein YTHDC2 Is a Potential Biomarker and Associated with Immune Infiltration in Head and Neck Squamous Cell Carcinoma. PeerJ 8, e10385. doi:10.7717/peerj.10385

Lu, L., Liu, L.-P., Zhao, Q.-Q., Gui, R., and Zhao, Q.-Y. (2021). Identification of a Ferroptosis-Related LncRNA Signature as a Novel Prognosis Model for Lung Adenocarcinoma. Front. Oncol. 11, 675545. doi:10.3389/fonc.2021.675545

Luan, T., Zhang, T. Y., Lv, Z. H., Guan, B. X., Xu, J. Y., Li, J., et al. (2021). The lncRNA ALMS1-IT1 May Promote Malignant Progression of Lung Adenocarcinoma via AVL9-mediated Activation of the Cyclin-dependent Kinase Pathway. FEBS open bio 11 (5), 1504-1515. doi:10.1002/2211-5463.13140 
Park, Y. P., Jin, L., Bennett, K. B., Wang, D., Fredenburg, K. M., Tseng, J. E., et al. (2018). CD70 as a Target for Chimeric Antigen Receptor T Cells in Head and Neck Squamous Cell Carcinoma. Oral Oncol. 78, 145-150. doi:10.1016/j. oraloncology.2018.01.024

Roh, J.-L., Kim, E. H., Jang, H. J., Park, J. Y., and Shin, D. (2016). Induction of Ferroptotic Cell Death for Overcoming Cisplatin Resistance of Head and Neck Cancer. Cancer Lett. 381 (1), 96-103. doi:10.1016/j.canlet.2016.07.035

Sung, H., Ferlay, J., Siegel, R. L., Laversanne, M., Soerjomataram, I., Jemal, A., et al. (2021). Global Cancer Statistics 2020: GLOBOCAN Estimates of Incidence and Mortality Worldwide for 36 Cancers in 185 Countries. CA A. Cancer J. Clin. 71 (3), 209-249. doi:10.3322/caac.21660

Wang, C., Li, Y., Jia, L., Kim, J. K., Li, J., Deng, P., et al. (2021). CD276 Expression Enables Squamous Cell Carcinoma Stem Cells to Evade Immune Surveillance. Cell stem cell 28 (9), 1597-1613. doi:10.1016/j.stem.2021.04.011

Wang, R., Zhao, L., Ji, L., Bai, L., and Wen, Q. (2019). Myocardial Infarction Associated Transcript (MIAT) Promotes Papillary Thyroid Cancer Progression via Sponging miR-212. Biomed. Pharmacother. 118, 109298. doi:10.1016/j. biopha.2019.109298

Wang, X., Li, H., and Shi, J. (2019). LncRNA HOXA11-AS Promotes Proliferation and Cisplatin Resistance of Oral Squamous Cell Carcinoma by Suppression of miR-214-3p Expression. Biomed. Res. Int. 2019, 8645153. doi:10.1155/2019/ 8645153

Xing, L., Zhang, X., and Chen, A. (2019). Prognostic 4-lncRNA-Based Risk Model Predicts Survival Time of Patients with Head and Neck Squamous Cell Carcinoma. Oncol. Lett. 18 (3), 3304-3316. doi:10.3892/ol.2019.10670

Yang, Y., Chen, D., Liu, H., and Yang, K. (2019). Increased Expression of lncRNA CASC9 Promotes Tumor Progression by Suppressing Autophagy-Mediated Cell Apoptosis via the AKT/mTOR Pathway in Oral Squamous Cell Carcinoma. Cell Death Dis 10 (2), 41. doi:10.1038/s41419-018-1280-8
Zhang, C., Xie, L., Liang, H., and Cui, Y. (2019). LncRNA MIAT Facilitates Osteosarcoma Progression by Regulating Mir-128-3p/VEGFC axis. IUBMB life 71 (7), 845-853. doi:10.1002/iub.2001

Zhang, Y., Mi, L., Xuan, Y., Gao, C., Wang, Y. H., Ming, H. X., et al. (2018). LncRNA HOTAIRM1 Inhibits the Progression of Hepatocellular Carcinoma by Inhibiting the Wnt Signaling Pathway. Eur. Rev. Med. Pharmacol. Sci. 22 (15), 4861-4868. doi:10.26355/eurrev_201808_15622

Zhou, R.-S., Zhang, E.-X., Sun, Q.-F., Ye, Z.-J., Liu, J.-W., Zhou, D.-H., et al. (2019) Integrated Analysis of lncRNA-miRNA-mRNA ceRNA Network in Squamous Cell Carcinoma of Tongue. BMC cancer 19 (1), 779. doi:10.1186/s12885-0195983-8

Conflict of Interest: The authors declare that the research was conducted in the absence of any commercial or financial relationships that could be construed as a potential conflict of interest.

Publisher's Note: All claims expressed in this article are solely those of the authors and do not necessarily represent those of their affiliated organizations, or those of the publisher, the editors, and the reviewers. Any product that may be evaluated in this article, or claim that may be made by its manufacturer, is not guaranteed or endorsed by the publisher.

Copyright (c) $2022 \mathrm{Li}$, Wang, Xiang and Chen. This is an open-access article distributed under the terms of the Creative Commons Attribution License (CC $B Y)$. The use, distribution or reproduction in other forums is permitted, provided the original author(s) and the copyright owner(s) are credited and that the original publication in this journal is cited, in accordance with accepted academic practice. No use, distribution or reproduction is permitted which does not comply with these terms. 